\title{
Politologie d'une rébellion. Une « gouvernance par la violence » au Nord de la Côte d'Ivoire?
}

\section{Michel Galy}

\section{OpenEdition}

1 Journals

\section{Édition électronique}

URL : http://journals.openedition.org/conflits/2208

DOI : 10.4000/conflits.2208

ISSN : $1777-5345$

Éditeur :

CCLS - Centre d'études sur les conflits lilberté et sécurité, L'Harmattan

\section{Édition imprimée}

Date de publication : 1 mai 2007

Pagination : 137-155

ISBN : 978-2-296-03084-8

ISSN : 1157-996X

\section{Référence électronique}

Michel Galy, «Politologie d'une rébellion. Une « gouvernance par la violence » au Nord de la Côte d'Ivoire? », Cultures \& Conflits [En ligne], 65 | printemps 2007, mis en ligne le 04 janvier 2010, consulté le 30 mars 2021. URL : http://journals.openedition.org/conflits/2208; DOI : https://doi.org/10.4000/ conflits.2208 


\title{
Politologie d'une rébellion.
}

\section{Une " gouvernance par la violence» au Nord de la Côte d'Ivoire?}

\section{Michel GALY}

Michel Galy est politologue, chercheur an Centre d'Etudes sur les Conflits (Paris).

$A$ mnesty international, qu'on ne peut suspecter d'excessive sympathie envers le camp gouvernemental ${ }^{1}$, décrit ainsi le massacre des gendarmes de Bouaké et de leurs familles, selon des témoignages recoupés :

\begin{abstract}
"A Bouaké, le 6 octobre 2002, une soixantaine de gendarmes accompagnés d'une cinquantaine de leurs enfants et de quelques autres civils ont été arrêtés dans leur caserne par des éléments armés du Mouvement patriotique de Côte d'Ivoire (MPCI) qui avaient pris le contrôle de la deuxième ville du pays depuis le 19 septembre 2002. Ces personnes ont été conduites à la prison du camp militaire du $3^{\mathrm{e}}$ bataillon d'infanterie. Ce même soir, des éléments armés du MPCI sont entrés à plusieurs reprises dans la prison et ont tiré en rafales, tuant et blessant des dizaines de détenus. Les survivants sont restés deux jours avec les blessés et les cadavres en décomposition sans recevoir de nourriture. Certains ont été contraints de transporter les cadavres et de les enterrer dans des fosses collectives et une dizaine d'entre eux ont très vraisemblablement été tués sur les lieux mêmes du charnier après qu'ils eurent enterré leurs camarades ».
\end{abstract}

1. Amnesty international encadre un compte rendu partiel - gardé un temps sous silence - du massacre de Bouaké, par des rappels du "charnier de Yopougon ", à Abidjan; procédé que l'on peut juger curieux, même si les exactions s'exercent bien par système et par cycles de vendetta (voir le rapport AI : Côte d'ivoire, de Youpougon à Bouaké, une suite de crimes impunis, 27 février 2003). Inversement, il est bien évident que la politologie de la violence au Nord et l'analyse de la rébellion ne préjugent pas des formes de pouvoir et des violences extrajudiciaires au Sud. (Voir notre analyse qui replace ces violences dans leur généalogie: Galy M., «La violence, juste avant la guerre ", Afrique contemporaine n²09, printemps 2004, pp. 117-140). 
Pas d'images de ces violences extrêmes, un peu à l'instar de Kigali : bien que les situations soient très différentes, la médiatisation saisit parfois, a posteriori, l’inverse du réel...

En revanche, l'opinion publique internationale se souvient de l'épisode télévisuel montrant, aux accords de Linas Marcoussis, le «leader des Forces nouvelles », Guillaume Soro, ex-activiste étudiant pressenti par le gouvernement français, en 2002, comme... «ministre de la Défense » à Abidjan! Quelle scène alors retenir pour symboliser une rébellion pour laquelle les médias occidentaux ont longtemps eu les yeux de Chimène, ces «rebelles qui sourient 2 », défenseurs des opprimés ?

L'analyse d'une telle rébellion semble d'autant plus une mission impossible que les observateurs sont le plus souvent des acteurs impliqués, ne seraitce qu'à cause des liens étroits qu'entretiennent historiquement les pays concernés - au-delà des affinités en réseaux politiques ou intellectuels, où se sont constituées des élites transnationales franco-africaines.

Pourtant, après le reflux d'un temps des illusions médiatiques sur les «sympathiques » rebelles nordistes, les campagnes de mise sous tutelle du camp gouvernemental par les institutions internationales et les massacres de novembre 2004 constituent bien des éléments majeurs dans le champ politologique africaniste et dans celui des relations internationales. Quelles sont donc les différentes phases de la connaissance de la rébellion ivoirienne et de ses perceptions médiatiques et politiques, aussi bien à Abidjan qu'à l'étranger ? Chacun comprend bien d'ailleurs que les réponses ne peuvent qu'être provisoires : les commanditaires, les financements, les alliances de la rébellion ne sont en effet connus que partiellement.

\section{Construction des rébellions : des périphéries insurgées ?}

Si les rebellions locales ont tout intérêt, pour asseoir leur légitimité, à se poser dans un contexte national et à nier les influences ou alliances extérieures, un comparatisme rapide découvre au contraire bon nombre de liens étonnants, au-delà des discours. Si des chercheurs comme Stephen Ellis et surtout Paul Ritchards ${ }^{3}$ ont mis l'accent sur les catégories d'âge et l'ethnicité de rébellions socialement et politiquement dominées, nous avons mis en évidence ${ }^{4}$ le caractère spatialement périphérique (dans une double acception : spatiale et sociale) de ces mouvements; remarque d'ailleurs assez désespérante à terme,

2. Expression qui fait florès même hors de l'Hexagone : voir par exemple «Ivory Coast, rebels with a smile ", BBC Focus on Africa, janvier-mars 2003.

3. Richards P., Fighting for the Rain Forest, Oxford, James Currey, 1996 ; Ellis S., The Mask of Anarchy: The Destruction of Liberia and the Religious Dimension of an African Civil War, Londres, Hurst, 1999.

4. «Les espaces de la guerre ", Hérodote, $\mathrm{n}^{\circ} \mathrm{III}, 4^{\mathrm{e}}$ trimestre 2003, pp. 41-56. 
car à cause de la conquête de l'époque coloniale, procédant des côtes vers l'intérieur, ce caractère politique des périphéries dominées et délaissées semble évidemment structurel.

Des chercheurs comme Claude Raffestin ${ }^{5}$, comparant les modes de construction de l'Etat, n’ont pas hésité à généraliser ce phénomène : quand l'Etat se montre plus fort et plus juste en son centre et au contraire plus distant - mais plus coercitif - dans des frontières floues ou disputées. Pour autant, un guérillero ne naît pas tout armé à chaque frontière, même en Afrique... Il y faut des circonstances particulières, une histoire propice, des financements, une organisation, etc. ${ }^{6}$. Si ce n'est pas le lieu d'une théorie générale des guérillas périphériques, on a pourtant trop dit que, calquées sur des Etats patrimoniaux, celles-ci étaient sans idéologie comme sans éthique, poussées par une primaire soif de prédation, selon les thèses caricaturales et économicistes d'un Paul Collier ${ }^{7}$ prêtant aux « War Lords » des mentalités uniquement entrepreneuriales et des mours seulement capitalistiques.

Le discours structurant des rébellions peut avoir une certaine consistance idéologique - bien que leurs pratiques en diffèrent sensiblement : discours anti-corruption et anti-crio ${ }^{8} \mathrm{du}$ RUF de Sierra Leone, revendications libératrices du NPLF 9 de Taylor contre la dictature Doe, revendications proRDR 10 ou illustrant des revendications nordistes contre l'ivoirité de la part MPCI en Côte d'Ivoire. Autant de registres évocateurs de revendications politiques, même s'ils heurtent l'épure marxiste des guérillas anciennes.

A travers ces idéologies mobilisatrices se dessinent d'ailleurs mieux les failles et les faillites des Etats concernés que les solutions à venir : fonction tribunitienne des guérillas devant la corruption du système électif, qui se heurtent aux structures sociales (que faire contre la démographie galopante et la subordination structurelle de la jeunesse ?). Elles ne proposent au mieux qu'une alternance de prédation : "à chacun son tour... de manger ! ", selon une métaphore bien connue, depuis les travaux désormais classiques de Jean-François Bayart, et qui exprime bien une certaine logique autochtone du politique.

5. Raffestin C., Pour une géographie du pouvoir, Paris, Litec, 1980.

6. Il y a certes une dynamique des rebellions qui n'est pas sans surprises contrastées : notamment ces guérillas « latentes », sur bien des frontières et des zones interstitielles, facilement manipulables ou réactivées, dont le plus célèbre exemple demeure l'épopée d'un Kabila végétant au temps de Che Guevara, jusqu’à son éphémère apogée aux sommets de l'Etat congolais. Voir l'ouvrage classique répertoriant ces divers mouvements : Clapham C., African Guerillas, Oxford, James Currey, 1998.

7. Collier P., Economic Causes of Civil Conflict and their Implications for Policy, Banque mondiale, 2000 ; Collier P., Hoeffler A., On the Incidence of Civil War in Africa, Banque mondiale, 2000.

8. Les crios forment la caste dominante de Freetown.

9. National Patriotic Front of Liberia.

10. «Rassemblement des Républicains » de l'opposant Alhassane Ouattara. 


\section{Le RDR a t-il créé un monstre ?}

Telle est en effet la « thèse moyenne » du coup d'Etat, intermédiaire entre la « théorie du complot » ex nibilo (dont le maitre, bien sûr, est encore caché) qui, universellement, satisfait à peu de frais les esprits simples, et celle du « reflet », qui verrait bien à tort les mouvements insurrectionnels comme le seul bras armé du RDR et de ses alliés. S'il faut la caricaturer à son tour en la dénommant, l'interprétation par "l'apprenti sorcier» comporte en effet l'avantage provisoire de rendre compte de l'autonomisation partielle de la rébellion et de son ancrage territorial, l'un expliquant partiellement l'autre sans toutefois en épuiser le sens.

Il faut donc partir méthodologiquement de ce «mystère des origines » qui n'est pas sans rappeler celui des sociétés secrètes, et dont le charme agit encore pour attirer de nouveaux combattants, aventuriers des temps modernes de toute la région, qui ont trouvé dans le nomadisme guerrier une situation durable... avant leur fin possible.

Les interprétations extrêmes et symétriques abondent : pour le camp présidentiel, il s'agit à la fois de putschistes militaires assoiffés de pur pouvoir, de «terroristes islamistes »- de type GSPC 11-, de cinquième colonne burkinabé, ou d'un simple «montage » des services occidentaux en général, français en particulier... Parfaitement contradictoires, ces interprétations ont le mérite d'une fausse clarté et, pourtant, s'appuient tour à tour sur d'indéniables indices tirés de la chronologie du mouvement.

Par certains côtés, le MPCI est bien une rébellion post-moderne, sauf à ignorer que bien des mouvements politico-militaires jouent des effets d'images et de signifiants ${ }^{12}$. On discerne facilement des composantes assez mal hiérarchisées et en constante réorganisation. Au tout début, deux groupes de jeunes recrues ivoiriennes «déflatées», de leur nom de classe: «Zinzins et Blofoués 13 » avec des rescapés en exil au Burkina de putsch et d'épurations ivoiriennes successifs, et parmi eux des proches - civils ou militaires - du leader du RDR.

11. Groupe salafiste pour la prédication et le combat.

12. Cette guerre connaît d'ailleurs des trouvailles linguistiques d' « agit-prop » militaro-politique qui laissent rêveur : à Marcoussis, les rebelles se sont dénommés « Forces nouvelles », les milices sudistes «Front de libération », les troupes françaises dénommées «impartiales » et leurs lieux de stationnement : «zones de confiance ", et le comité de tutelle : "Groupe de travail international »; contre les «collaborateurs », les « résistants » et les «patriotes » se disent soucieux de « refondation »... Le conflit est aussi sémantique et dans cette imagination terminologique débordante, bien des crises et des projections semblent se rejouer.

13. Environ 750 jeunes militaires promis à la démobilisation en décembre 2002, et base sociale restreinte des putschistes (eux-mêmes formés de strates successives de déserteurs de l'armée après des coups d'Etat manqués, ou chassés de force, après des émeutes sanglantes, en faveur d'un régime civil et pour la reconnaissance de l'élection de Laurent Gbagbo, en octobre 2002). 
Au-delà, foisonnent des données plus hypothétiques : si le lien avec des mouvements estudiantins et «l'école de la Fesci ${ }^{14}$ » est évident avec Guillaume Soro, si des militaires de l'armée officielle se sont ralliés (à mesure, pour certains, qu'ils étaient limogés à Abidjan), la présence au début de la rébellion de «mercenaires » libériens et/ou sierra léonais semble attestée et fait partie de ce « nomadisme » des guerriers ouest-africains.

A ce propos, les «guerriers urbains» d'Abidjan magnifiés par la cinéaste Eliane de la Tour ${ }^{15}$ sont-ils représentatifs des milieux délinquants urbains qui auraient pu passer à la rébellion nordiste ? Si les cas avérés semblent minoritaires, il est en revanche certain que marginaux et jeunes exclus (qui forment en partie des unités d'« enfants soldats », surtout vers l'Ouest libérien), petits métiers, chômeurs des villes et des campagnes du Nord ont rapidement formé des troupes supplétives aux soldats d'une rébellion, au début inférieure à un millier d'hommes - et qui a trouvé à travers eux un certain enracinement ethnique. Plus hypothétique encore ${ }^{16}$, la présence de contingents burkinabés, avec ou sans uniforme, même s'il est de notoriété publique que Ouagadougou sert de base arrière aux leaders rebelles qui y possédaient, grâce au président Compaoré, villas et $4 \times 4$, subsides et armes, ainsi que des facilités de voyage à l'étranger et d'entraînement militaire.

De ces contingents hétérogènes, sigles et médias ont trop vite fait une unité, à la fois guérilla et mouvement politique. L' «opération MPCI » a bien été, de l'avis de beaucoup, montée a posteriori, après l'échec du putsch et son cantonnement par la force Licorne au Nord de la Côte d'Ivoire. « Force du signifiant 17 » : à l'époque, ni le sigle ni a fortiori la fonction de son « Secrétaire général » n'avaient d'existence, ni de consistance : s'il ne s'agit pas de pure « vitrine » sémantique, ni d'intoxication de la presse internationale, l'opération a introduit des réalignements inédits, mais a favorisé un clivage inattendu militaires/civils, ces derniers dirigeant par le Verbe, source de constantes tensions. Des opérations secondaires, comme la duplication d'un MPIGO ${ }^{18}$ ou du MJP 19 à l'Ouest de la Côte

14. Voir l'article de Konaté Y., «Les enfants de la balle », in Vidal C. (dir), Côte d'Ivoire, l'année terrible, Paris, Karthala, 2002.

15. Notamment les films : Bronx barbes, 2000 ; Les Oiseaux du ciel, 2006 ; voir aussi : « Les ghettomen : étude des processus d'identification par l'illégalité à travers les gangs de rue à Abidjan et San Pedro ", Actes de la recherche en sciences sociales, Paris, 1999, n²129, pp. 68-84

16. Question clef opposant les autorités ivoiriennes qui demandaient en septembre 2002 les applications des «accords de Défense » devant «l'agression extérieure », contre le ministère français de la Défense qui y voyait une affaire «ivoiro-ivoirienne». Sur ce point, les récentes interventions au Tchad et en République centrafricaine ont plutôt confirmé que cette distinction était caduque, et les fameux accords, à géométrie variable...

17. Voir les toujours actuelles réflexions de Jacques Berque sur la puissance des «emblèmes onomastiques » dans la définition des groupes: "Qu'est ce qu'une tribu nord-africaine ? ", in Hommage à Lucien Febvre, Armand Colin, Paris, 1953. Ce pouvoir de la nomination se retrouve dans les «noms de guerre » qui s'appuient sur des traditions autochtones mixées à la sous-culture des séries B occidentales ou du Kung-Fu.

18. Mouvement populaire ivoirien du grand Ouest.

19. Mouvement pour la justice et la paix. 
d'Ivoire ${ }^{20}$, ont tenu le temps de délégations surréalistes à Marcoussis et ont été réduites par la force, de manière sanglante... par la direction rebelle elle-même!

Le pouvoir rebelle, en dehors du clivage factionnel I.B./Soro, est plus à concevoir comme une nébuleuse, faite d'agencements changeants de chefs de guerre et de leurs troupes ${ }^{21}$, que comme un système de commandement hiérarchisé. Ici aussi, la labellisation des leaders locaux peut faire illusion, tels les « coms zone» («commandant de zones », zones qui sont elles-mêmes divisées en « commandements opérationnels », puis en « postes »- organisation largement fictive...) ; mais la pratique semble celle de rivalités incessantes pour les rackets, régulées par les armes. Ainsi, une logique de fiefs se développe, comme une territorialisation de la violence, obligée par là même de passer des alliances avec les pouvoirs autochtones, comme Koné Zackaria, le chef de guerre de Vavoua. Un certain repli sur les fiefs se fait jour, depuis l'exécution du caporalchef Bamba Kassoum, taxé de pro-I.B. et bien avant, celle du chef de guerre Adams, à Korhogo.

Cependant, le corps des «dozos», proche des kamajors sierra léonais, reste une force intermédiaire entre les anciens militaires ivoiriens, gardes du corps ou mercenaires, et la piétaille des milices villageoises ou urbaines, leurs troupes de base. A mi-chemin entre une tradition poro réinterprétée et une armée rebelle, les dozos ne sont aucunement l'apparition au grand jour des "chasseurs traditionnels villageois", comme le montre bien Marianne Ferme ${ }^{22}$, dans le cas sierra léonais. Toutefois, leur attirail et leurs pratiques magico-religieuses, qui s'inspirent de l'univers poro, ne sont pas sans influence, militairement efficaces car socialement partagés.

Sur le savoureux site Internet de la « Compagnie guépard », autrement dit les dozos intégrés dans la rébellion à Bouaké, un journaliste sympathisant (et complaisant : ne sont-ils pas les "guerriers de la Lumière » ?) détaille leurs

20. «Mouvements fantômes », que des analystes férus de « renseignement militaire » essayaient en vain d'estimer dans leurs effectifs, équipement militaire et organigramme, alors qu'ils étaient dans un premier temps des émanations médiatiques du MPCI (mais avec des effets de sympathies ethnicistes auprès du peuple Dan proches de l'ex-président Guei) et étaient devenus la dénomination de factions libériennes émigrées - comme chez leurs adversaires loyalistes d'ailleurs (voir les rapports de Global Witness sur ce point).

21. Rapports de pouvoir et de situations factionnelles, d'alliances politiques ou ethniques que les expressions toutes faites de «balkanisation » entre «seigneurs de la guerre » ne résument pas, fussent-ils rebaptisés « War Lords »...

22 . Ferme M., "La figure du chasseur et les chasseurs-miliciens dans le conflit sierra-leonais », Politique africaine $\mathrm{n}^{\circ} 82$, juin 2001 . A contrario, voir par exemple le rapport du « collège interarmées » de l'Ecole militaire de Paris, dont le titre dit tout : «Lorsque la tradition menace la sécurité » (J. Remarck) qui précise que chez les «dozos ou donzons» de Côte d'Ivoire, en février 1999, "plus de 40000 chasseurs ont été recensés » (ce qui est fort exagéré); constat plus inquiétant, "ils possèdent plus de 33000 armes modernes ", et, précise l'auteur «surtout des calibres 12, sans aucun permis de port d'armes »(!). On peut préférer les recherches savantes de l'anthropologue russe Vladimir Arseniev, qui connaît bien les dozos maliens : «Un groupe social particulier », Etudes maliennes n³, Bamako, 1980. 
pouvoirs : transformation en animaux, invisibilité, invulnérabilité aux balles, et prescience pour les « chefs dozos », comme Bamba, Ibrahim Konaté, Chérif Ousmane! On comprend que les combattants sudistes soient surclassés... Comme au Libéria, l'aspect emprunte à la fois à la «panoplie ethnique»du mysticisme des chasseurs : dreadlocks, kaolin, attirail de miroirs, colliers de cauris, amulettes et tuniques sont effectivement censés terrifier l'adversaire.

Bien que, globalement, un appareil de conquête se substitue à l'appareil d'Etat détruit et se surimpose aux communautés autochtones (en particulier en pays baoulé, où la rébellion est bien plus « étrangère » qu'au Nord sénoufo ou malinké), d'autres dispositifs s'observent sporadiquement. Au Nord, rien ne peut fonctionner au quotidien sans les pouvoirs autochtones, comme ceux des «tarfolo » sénoufo pour la terre, ou des « grandes familles » comme les Gbon Coulibaly à Korhogo. A plusieurs reprises, une articulation avec le «pouvoir coutumier » a évité à la rébellion d'être décimée : en particulier lors de « l'épuration ethnique » de Bouaké (exclusion des populations baoulé au profit des Dyoula) et lors des tentatives, en octobre 2004, pour reprendre la ville, lorsque des vendetta et des règlements de compte entre groupes ethniques prenaient de l'ampleur. Des négociations et des médiations ont empêché in extremis la situation de passer hors de contrôle de la rébellion, de même, lors de massacres réciproques entre partisans de I.B. et de Soro en décembre 2003.

\section{Pratiques de la violence}

La violence militaire, criminelle ou politique, en zone rebelle, est mal connue et mal documentée, à tel point que certains observateurs, paraphrasant la formule connue en Occident, prétendent que les «observateurs des droits de l'Homme sont au Sud, les crimes de guerre au Nord». En termes de gouvernance et de définition de la légitimité de la rébellion, de la cohérence de ses pratiques avec son idéologie, le sujet est pourtant crucial.

Le thème de la violence peut cependant se nuancer selon les temps et les lieux : dans sa dimension chronologique, les débuts sont marqués non seulement par la «violence de guerre » contre l'armée ivoirienne, et les autres «corps habillés», mais aussi par des massacres de fonctionnaires et de civils sudistes qui restent à préciser ; puis, par une «épuration ethnique » largement sous-évaluée, notamment de la part de la galaxie humanitaire qui collabore avec la rébellion en zone nord, en particulier dans la ville de Bouaké; enfin, par un massacre ethnico-factionnel au sein de la rébellion, lors de l'épuration violente par les miliciens de Guillaume Soto - surtout sénoufo, des partisans d'Ibrahim Coulibaly - en grande partie malinké :

\footnotetext{
" "L'Opération des Nations unies en Côte d'Ivoire" (ONUCI) a découvert trois charniers au Nord de la Côte d'Ivoire, dans la région
} 
de Korhogo où se sont rendus par deux fois ses enquêteurs chargés des droits de l'Homme et de la police, du $1^{\mathrm{er}}$ au 12 juillet et du 22 au 26 juillet derniers. Avant même la publication de leur rapport, les spécialistes onusiens ont annoncé le 2 août qu'ils avaient pu établir la mise à mort, par balles, décapitation ou asphyxie, de quelque 99 personnes au moins. Les corps ensevelis dans trois sites seraient identifiés comme ceux de victimes des affrontements qui ont opposé dans la métropole nordiste des factions rivales de l'ancienne rébellion du Mouvement patriotique de Côte d'Ivoire (MPCI), les 20 et 21 juin dernier. La victoire était alors revenue à Guillaume Soro, l'actuel chef politique des Forces nouvelles 23 ».

Cela n'empêche pas une «violence ordinaire» contre la population autochtone, régulière et sanglante, encore plus mal connue, due en particulier à l'absence de forces de l'ordre de l'appareil militaire rebelle ${ }^{24}$ et qui devrait conduire à reposer, comme au Congo, en Ouganda, au Libéria, au Sierra Leone, etc., la question de la criminalisation de ce pouvoir et des sanctions juridiques encourues.

A ce propos, une sorte de scène prototypique pour l'opinion ivoirienne pourrait être celle du massacre gratuit, par un groupe de rebelles, de jeunes filles baoulé exécutant dans un petit village près de Sakassou une danse rituelle d'exorcisme de la violence. Cet épisode mineur, qui a autant touché les imaginations que l'immolation des enfants de gendarmes sudistes de Bouaké, a bien sûr à voir avec la perte d'une certaine innocence du vivre ensemble, du temps des rituels remplacé par celui de la violence pure.

Enfin, ce que l'on a pu appeler la « libérianisation » de l'Ouest conduit à des formes de violence plus anomiques, en tout cas proches de celles observées lors du conflit du Libéria, à tel point que la rébellion elle-même s'est débarrassée de leaders comme le «pseudo Doe » et de groupes nomades enfreignant les pratiques de la rébellion de Bouaké, elle-même pourtant peu regardante sur les exactions contre les civils.

Ainsi on a pu parler d' "épuration ethnique sporadique » sur plusieurs points de la zone rebelle, en particulier dans les territoires proches du Libéria, contre les Guéré en particulier. Il est vrai que cette « libérianisation » du territoire ivoirien reste partagée, puisque les deux camps - loyaliste et rebelle - ont instrumentalisé à la fois des couples d'oppositions ethniques transfrontalières, depuis longtemps sous-jacents (Gyo/Dan vs Wê/Krahns) et des groupes nomades mercenaires, issus des conflits du Libéria et de la Sierra Leone, et en

23. Monique Mas, sur RFI, le 03 août 2004.

24 . Y compris, à l'inverse pour un « maintien de l'ordre » très militaire : en 2003 par exemple, pillage et racket étaient parfois punis par des exécutions capitales sur la grand place de Bouaké. 
quelque sorte recomposés pour poursuivre leurs carrières guerrières. L'évolution sanglante de l'Ouest a tellement porté tort à l'ensemble de la rébellion, que le "pseudo Doe », leader éphémère de l'inconsistant MPIGO, a vraisemblablement été exécuté par les partisans de Guillaume Soro ${ }^{25}$.

Dans la «capitale rebelle », Bouaké, il semble que les pratiques des groupes militaro-mafieux, de type «Camorra», «Cosa nostra» et autres «Ninja ", qu’a connu Abidjan sous la dictature militaire du général Gueï, restent les mêmes. Cela n'étonne aucun analyste, tant il est connu qu'un groupe des «fondateurs » de la rébellion est justement issu de cette mouvance.

Selon une enquête de la Ligue des droits de l'Homme ivoirienne (LIDHO) de février 2003, «environ $80 \%$ des violences sont perpétrées par les rebelles » : il faut bien dire que l'image qu'en donnent les médias français et une certaine recherche politologique ne l'a guère évoqué... Comme nous l'avons signalé, les enquêtes villageoises en particulier restent à faire dans la zone nord. Depuis plusieurs années, les ONG françaises de terrain, telles $\mathrm{ACF}$, remarquent que la violence systématique contre les populations civiles de l'Ouest sont très sous-estimées. Il faut y voir la conjugaison d'incursions libériennes et de pratiques extrêmes, qui échappent en partie au (contre-)pouvoir de Bouaké, avec une criminalisation des forces en présence, aggravée par un système de représailles non seulement interethniques (inter-ivoirien), mais avec les immigrants nordistes au sens large («dyoula» sahéliens) dans une compétition foncière aiguë.

\section{Idéologie mandingue et revendications ethnico-politiques}

Si le sujet n'était pas tragique, il serait savoureux d'observer combien, une fois de plus, un certain engouement des médias occidentaux, de savantes manipulation et corruptions de l'opinion par les affidés de la rébellion, l'attente jamais démentie par les faits de militants tiers-mondistes de bonne foi d'une «Cause », enfin juste, de «libération des peuples», complexe d'actions et d'attentes qui ont joué dans la perception de la rébellion ivoirienne. Car, à la lumière des pratiques observées, ce petit «Timisoara exotique 26 » est-il bien différent - hors son échelle - des scandales cambodgiens, iraniens, chinois ou soviétiques dans lesquels beaucoup se sont successivement fourvoyés?

Pour distinguer les strates de l'idéologie de la rébellion ivoirienne, on peut s'intéresser, au-delà des idées professées, aux idéo-logiques locales, replacées

25. D’origine baoulé, N'dri N’Guessan alias sergent Félix Doe, a été tué en avril 2003, quand la direction du MPCI a liquidé les miliciens libériens proches de Sam Bockarie ; sa « carrière » comprend aussi sa participation aux accords de Linas Marcoussis...

26. Voir en particulier la vision critique d'Acrimed sur la désinformation à Paris : Journalisme de guerre : retour sur la "crise ivoirienne » de novembre 2004, août 2005. Schneidermann D., « En Côte-d'Ivoire, le journalisme en uniforme », Libération, " Médiatiques », 12 novembre 2004. 
dans la longue durée braudélienne, mais aussi distinguer les représentations spécifiques des factions et groupes qui la composent.

Comme on l'a vu, rien ne destinait Guillaume Soro, leader étudiant en rupture de ban, proche du FPI puis du RDR ${ }^{27}$, à régner en caudillo sur les savanes du Nord ivoirien. Son but, comme celui de tout jeune Turc local, et des cadets sociaux en général, était le pouvoir dans la capitale, monopolisé par une caste politique d' 'Anciens » dont il ne pouvait être qu'un représentant commode, un pouvoir vicariant, comme diraient les socio-analystes. En revanche, la problématique de "discrimination» envers les Sahéliens au sens large, les Nordistes en particulier, est socialement juste, dans une certaine mesure, bien qu'elle s'accompagne d'une volonté d' " hégémonie dyoula » que les partisans locaux ou occidentaux de la rébellion se gardent bien de mettre en avant.

Car la «conquête du Sud » est en effet à replacer dans un trend de longue durée ${ }^{28}$, de descente des peuples sahéliens vers les zones côtières, mêlant « migrations de guerre » et expansion économique. Et la difficile question de l'islam en Côte d'Ivoire ${ }^{29}$ complique les choses - même si l'interprétation du conflit en termes de « guerre de religions » (si ce n'est en termes de conflits de « civilisation» pour les huntingtoniens téméraires) n'est pour le moment du moins qu'une interprétation excessive et très militante à l'étranger du pays. Inverse et symétrique de l'«ivoirité 30 », fondée théoriquement par le régime d'Henri Konan Bédié mais portée par un mouvement de fond à la fois xénophobe et nationaliste depuis l'ère houphouétiste, l'idéologie mandingue se réclame d'une domination sahélienne large, s'inspire d'une hégémonie politique de longue durée, légitime des pratiques contemporaines de discrimination à rebours qui ont conduit aux pires violences.

$\mathrm{La}$ «poudrière identitaire nordiste » est en effet à replacer tout d'abord dans un complexe culturel très large qui, à travers des sociétés forestières et sahéliennes, comprend des caractéristiques communes, à travers la « gouvernementalité poro » et une surimposition de pratiques islamiques bien plus récente. Bien que ces aires, comme celles de l'empire mandingue et de la mouvance de Samory Touré, ne se recoupent pas, la doxa mandingue les mêle dans

27. Notamment colistier d'Henriette Diabaté, n² du RDR, aux législatives de 2002, à PortBouet. Notons qu'à l'inverse, un ancien du FPI, Louis Dacoury-Tabley, est d'autant plus mis en avant qu'il s'agit d'un ancien proche de Laurent Gbagbo.

28. Voir notre contribution au colloque d'Abidjan du "Centre de recherche sur l'Etat en Afrique ", en juillet 2006, sur les « Migrations de guerre » (actes du colloque à paraître chez l'Harmattan en avril 2007) qui vise notamment à rompre avec une imagerie idéalisée des flux transfrontaliers.

29. Voir l'ouvrage récent de Miran M., Islam, histoire et modernité en Côte-d'Ivoire, Paris, Karthala, 2006, qui n'étudie pas toutefois la sociologie de l'islam en pays rebelle, le terrain étant bien sûr quasi impossible.

30. Voir l'analyse de C. Sandlar, "Les “titrologues” de l'ivoirité », Outre terre n¹1, juin 2005, et l'ouvrage fondateur d'un philosophe ivoirien : Boa Thiémélé R. L., L'Ivoirité entre culture et politique, Paris, L'Harmattan, 2003. 
une revendication de supériorité passée et de destinée manifeste à venir, tandis que le regrettable présent démontre l'inverse : délaissement étatique et discriminations multiples. Dans une certaine mesure, l'idéologie mandingue fait bien sûr partie de ces revendications inversées de peuples sous le joug, mais rejoue aussi en mineur le mouvement de la négritude et la mythification du glorieux passé « égyptien » fondé par Cheik Anta Diop.

Des composantes plus récentes s'appuient, comme on l'a évoqué, sur la «descente multiséculaire » de la « révolution dyoula» qui mêle commerce et foi, migration vers les plantations et les capitales côtières et sentiment diffus de supériorité sur les "sauvages et païens forestiers », rebaptisés récemment du nom-stigmate de «bushis», comme antonyme de «dyoulas».

La pauvreté de la zone nord de la Côte d'Ivoire s'est effectivement combinée à une sujétion politique à l'ère houphouétiste, quand les Nordistes servaient de force d'appoint au PDCI-RDA - certains les ont d'ailleurs qualifiés de «bétail électoral»! La concentration des plantations, où les manœuvres nordistes connaissaient une sorte de «travail forcé » collectif (sur un mode à la fois capitalistique, lignager et communautaire qu'il serait trop long ici d'analyser), s'est combinée avec le caractère privilégié par les différents régimes du développement de la zone sud, et ce, avec l'évidente complicité des «bailleurs de fond » raisonnant en termes pseudo-économicistes de "Côte d'Ivoire utile » et, sans le dire, partant de leurs rapports de proximité avec le pouvoir ivoirien pour semer de «cimetières de projets » le pays baoulé.

Les peuples du Nord, sénoufo, lobi, malinké, etc., étant en continuité avec le Mali et le Burkina Faso, portant les mêmes patronymes de chaque côté des frontières très poreuses, ont effectivement subi, dans le Sud et en particulier à Abidjan, des pratiques discriminatoires en termes de contrôle, racket, pièces d'identité, notamment, bien sûr aggravées depuis la guerre pour des motifs de sécurité.

Les peuples du Sud, le dos à la mer, effrayés par les $30 \%$ de résidents non nationaux et par les quelques $50 \%$ de Nordistes dans la capitale, parfois minoritaires comme dans l'Ouest sur leurs terres ancestrales, ont vu jusqu'à leur existence menacée, et ont redouté à leur tour d'être victimes d'une domination politique nordiste. Rappelons ici qu'un des principes non écrits de l'idéologie houphouétiste se fondait sur une répartition des fonctions politicoethniques, où les Nordistes dominent jusqu'à aujourd'hui des pans entiers de l'économie, notamment le commerce Nord/Sud et les transports, tandis que les baoulés se sentaient responsables du pouvoir politique.

Cette situation de discrimination économique et de sujétion contemporaine a nourri l'idéologie mandingue d'apports marxisants, s'opposant à la domination des "peuples prolétaires", mais aussi des discours inspirés de 
revendications identitaires en termes de discriminations raciales et ethniques, reprenant une terminologie anti-apartheid, ou décalquant le vocabulaire de la communauté afro-américaine pour les droits civiques - exprimée ici, comme pour les natives libériens en lutte contre les congos de la capitale, par le refus d'une « citoyenneté de seconde zone ». Ce complexe de revendications idéologiques assez disparate, appuyé sur des accusations à vrai dire peu étayées de discrimination anti-musulmane, a pu contribuer à populariser l'idéologie mandingue et les revendications au-delà des frontières (d'où des sympathies diffuses que nous avons pu constater non seulement au Burkina Faso et au Mali, mais aussi au Libéria et en Sierra Leone), jusqu'à... l'extrême gauche européenne, dont les textes montrent une projection systématique du problème sur la situation des immigrés musulmans en Europe.

L'idéologie mandingue s'est cristallisée sur un «homme providentiel » (bien sûr Alhassane Dramane Ouattara), sorte de héros culturel, puis s'est davantage fragmentée sur le nom de plusieurs représentants et, enfin, a presque implosé après les affrontements fratricides de 2005, symptômes de réalités ethnico-régionales sous-jacentes, de fait très antagonistes.

\section{Un proto-Etat sahélien ?}

Dans les derniers temps (particulièrement en 2006), la rébellion s'est dotée d'embryons d'appareils d'Etat et d'attributs de souveraineté, qui font craindre à certains observateurs la partition de la Côte d'Ivoire et l'autonomisation d'une «République du Nord », vouée tôt ou tard à une réunification avec le Burkina.

Il est mal connu en revanche à l'extérieur du pays que le gouvernement légal d'Abidjan continue à soutenir à bout de bras certains secteurs techniques, à fonds perdus. Non seulement les fonctionnaires nordistes continuent à être salariés par Abidjan, mais eau et électricité sont fournis par les appareils techniques de l'Etat ivoirien à la zone rebelle, faveur d'autant plus appréciée qu'aucune facture n'est réglée.

Sous influence de médias ou de chercheurs sympathisants de la rébellion, un certain nombre de puissantes ONG occidentales se sont, depuis 2002, substituées à l'appareil d'Etat ivoirien détruit par la rébellion, à tel point que cette action fait actuellement débat dans la sphère humanitaire. C'est notamment le cas du secteur de la santé : tandis que les soins de santé primaire (infirmeries, centres de santé villageois,...) sont inexistants et qu'entre VIH, paludisme et maladies plus ordinaires, les villageois sont décimés, une ONG comme MSF salarie à Bouaké des dizaines d'expatriés et des centaines de «locaux », "alliés objectifs de la rébellion », intouchables et extrêmement populaires au Nord (et à l'abri des exactions et des brigandages, naturellement à minimiser par ces acteurs humanitaires). Cette ONG tient, à la limite, le rôle ambigu de «ministère de la Santé du MPCI »! Les organisations internatio- 
nales se prennent au piège de cette « aventure ambiguë » : ainsi l'Unesco a-telle financé le fonctionnement du système éducatif au Nord.

L'économie comprend deux secteurs tout à fait antagonistes et, à bien des égards, caricaturaux : l' «économie de prédation ", à la fois individuelle et collective, comprend le trafic informel de toutes les matières premières - coton, bois, cacao, sucre, diamants -, transports au profit des dignitaires de la rébellion et de leurs alliés régionaux, comme le Burkina et peut-être le Sénégal. Outre le mystérieux financement initial, le «casse de la BCEAO » de Bouaké ${ }^{31}$ (mais aussi à Korhogo et Man), en septembre 2003, a permis de rapides enrichissements personnels, bien que le changement rapide de monnaie ait entraîné certaines difficultés de blanchiment.

La rébellion semble prise dans un curieux dilemme : soit elle joue le jeu d'un gouvernement d'union nationale et participe de la vie politique ivoirienne, permettant le redéploiement de l'administration ; soit, pour répondre aux critiques de prédation et de non-administration manifeste du "Soroland », elle crée avec des appuis burkinabés sa propre administration. Mais, dans ce dernier cas, elle renforce l'issue autonomiste puis sécessionniste de la "République du Nord » (ou, plus couramment chez les rebelles, "Côte d'Ivoire Un ») ou de manière plus réaliste, irrédentiste, comme future province du "pays des hommes intègres ", si l'on peut encore employer cette expression, datant de Thomas Sankara, pour le Burkina. Ce dernier cas, sans doute refusé par la CEDEAO (Communauté économique des Etats de l'Afrique de l'Ouest) et l'UA comme un dangereux précédent, serait en revanche un prétexte au camp loyaliste pour organiser des élections internationalement reconnues, cantonnées à la zone sud.

C'est ainsi que la rébellion a entrepris un processus multiforme d'étatisation : ouverture de banques (Caisse d'épargne populaire et de crédit en Côte d'Ivoire CEPCCI), projets d'écoles de douane, gendarmerie, police, établissement d'une « radio télévision MPCI». Plus récemment, une « centrale » (sic!) permet, de manière minoritaire, de collecter une sorte d'impôt, que certaines sources estiment détournées massivement au profit des seigneurs de guerre rebelles.

Dans les « sommets de l'Etat » rebelle, on assiste à un curieux mimétisme de représentation avec l'Etat officiel, inversement symétrique aux pratiques de prédation continue ${ }^{32}$ : des chefs guerriers comme le fameux Wattao sont bombar-

31. De plus de 50 milliards de Fcfa, ce hold-up poussera la banque ouest-africaine à changer de billets, mais permettra à la rébellion d'assurer sa survie avec une application caricaturale des détournements par ses leaders vers des comptes à l'étranger.

32. Reconstruire les services de l'Etat est d'ailleurs incompatible avec des pratiques antérieures comme la destruction volontaire par les rebelles de l'état civil : de sorte que la délivrance de pièces d'identité et de cartes d'électeurs, débat brûlant et récurrent, s'avère aujourd'hui impossible. 
dés « chef d'état-major adjoint » (sic), tandis que le « président » GuillaumeSoro installe un «cabinet civil », des écoles de gendarmerie, police, douane, etc.

Un des paradoxes de la situation dans la zone nord semble la fuite continue de la population, conséquence d'une destruction non seulement des appareils administratifs, mais de l'économie elle-même. Un rapport de l'ONUCI, en janvier 2004 (à une époque où les rebelles, ne touchant plus de solde, terrorisaient la population), explique :

«La situation économique dans les zones tenues par la rébellion est sombre. La fermeture des entreprises et des banques, la quasi-disparition des services administratifs, la réduction draconienne des services sociaux, la perte de milliers d'emplois, partant, des moyens de subsistance, ont entrainé l'effondrement de l'économie informelle ».

En attendant qu'une esquisse de proto-Etat se développe, les populations pratiquent l'exode à grande échelle : les « réfugiés d'Abidjan » sont plus d'un million, sans compter ceux qui ont passé les frontières et se massent dans les « villages-frères » du côté gouvernemental. «Régner sur les cimetières » ou sur une zone exsangue ne motivant pas excessivement les rebelles, l'option de partage du pouvoir dans la capitale pourrait s'en trouver paradoxalement renforcée...

\section{Rébellion dans la rébellion et jeux d'alliances}

La scission violente de la rébellion prouve à la fois son enracinement ethnique, la faiblesse de ses revendications idéologiques, mais aussi, paradoxalement, ses capacités de survie et d'adaptation, notamment pour avoir continué à résister au camp loyaliste.

Coup d'Etat manqué monté à la fois de l'intérieur, et instrumentalisé par un Etat limitrophe, devenu rébellion et pourtant participant de manière fluctuante au gouvernement national, fonctionnant en système avec les mouvements nomades ouest-africains et passant des alliances régionales, visant le pouvoir à Abidjan en coordination avec la branche civile de l'opposition, en même temps établissant les prémices d'un proto-Etat du Nord, le mouvement rebelle apparaît très composite et prend des formes en apparence très contradictoires.

Pourtant, une anthropo-sociologie de la rébellion montre au contraire son double enracinement ethnique, son rôle à la fois tribunicien par rapport aux inégalités de développement et son exercice continu d'une violence sanglante envers les civils et ses propres membres. De fait, rapporté aux formes des autres rebellions ouest-africaines (ou plus lointaines, comme les mouvements congolais), il semble aussi confirmer les thèses de Richards sur sa fonction de mouvements $\mathrm{d}$ ' " entrepreneurs de la violence » - voie guerrière pour sortir les cadets sociaux de leur exclusion du social - et pour leurs leaders de leur sujétion, en tant que 
catégorie d'âge et intellectuels prolétarisés, au leadership politique représentatif. De même, leur situation périphérique explique à la fois son rôle de porte-parole des Nordistes ivoiriens exclus de la «Côte d'Ivoire utile» depuis le «temps colon », et ses accointances avec le régime burkinabé soucieux d'irrédentisme et de prédation économique, un peu à l'image du Rwanda envers les richesses congolaises. Puis les événements de 2004 dans la zone nord ont montré l'implosion du mouvement insurrectionnel, dès lors transformé au fond en deux « ethno-rébellions » correspondant aux groupes sénoufo, autour de Guillaume Soro, et malinké/dyoula (au sens restreint) se revendiquant d'Ibrahim Coulibaly, et, pour certains analystes militaires, son émiettement.

Loin d'être isolée et uniquement tournée vers Abidjan, la rébellion s'est tournée vers des alliances régionales et internationales mouvantes, participant de la « guerre nomade » ouest-africaine et du jeu des rivalités politiques classiques entre pays voisins, notamment de la zone CEDEAO. C'est dire au passage la «fausse bonne idée ", qui ne se limite pas à l'Afrique de l'Ouest, ni au continent, de la logomachie onusienne alliée à de sympathiques utopies panafricaines, de vouloir confier la « résolution des conflits » à des forces « régionales », à un « corps » ou « force d'intervention » de l'Union africaine. Ce raisonnement ubuesque feint d'ignorer les problèmes politico-militaires qui fâchent: voit-on sérieusement un contingent burkinabé faire la paix à Abidjan, tandis qu'un autre ferait la guerre à Bouaké ? Ou tire-t-on les conclusions des résistibles «interventions africaines ", dont la dernière et catastrophique en date a lieu en ce moment au Darfour?

Il est de notoriété publique que le leader du MPCI, Guillaume Soro, est l'heureux possesseur d'un passeport diplomatique sénégalais, qu'il possède des biens considérables dans sa base arrière de Ouagadougou et qu'il se déplace en France librement, pour faire la promotion médiatique de ses idées et de ses écrits. Ces exemples, caricaturaux mais bien réels, montrent bien le sens des alliances de la guérilla.

En effet, la « guerre nomade » amplifie de manière entropique des phénomènes d'instrumentalisation réciproque des oppositions civiles et armées, des mouvements de guérillas et des factions politiques, entre gouvernements africains antagonistes. Le cas le plus connu est certainement l'opposition d'école entre le président Houphouët Boigny de Côte d'Ivoire et Sékou Touré de Guinée, dont tous les «coups d'Etat » n'étaient pas imaginaires, même si la plupart n'étaient que des fictions propres à de sanglantes répressions.

Outre l'instrumentalisation par les deux camps des groupes armés libériens, à l'appui de la guérilla par le Burkina - et plus discrètement par le Mali et le Sénégal - le camp loyaliste peut se prévaloir de l'alliance avec l'Afrique du Sud, le Rwanda et l'Angola (et au-delà, de la Chine et de la Russie) - qui esquissent une redoutable « ligue anti- française » en relations internationales, 
reprochant à l'actuel régime en place à Paris des actions de déstabilisation néofoccardiennes, mêlant pressions financières, diplomatiques, militaires et médiatiques savamment orchestrées, mais parfois globalement inefficaces.

Comment qualifier la «gouvernance rebelle » et son système politique ? De toute évidence, l'écart est extrême entre un discours généreux de « Robins des bois » égalitaristes ou de « Freedom fighters » nordistes, et les pratiques de violence discontinue. Contrairement au camp gouvernemental, on ne voit guère en zone rebelle de contre-pouvoirs, ni de système électif. Pire, aucun appareil d'Etat, technique en particulier, ne gère le quotidien : si ONG et système onusien se sont curieusement substitués aux administrations chassées de la zone sud, c'est qu'il est de notoriété publique que, par exemple, une terrible surmortalité sévit, tandis que la criminalité et les exactions explosent.

Une comparaison éclairante à qui a connu le Libéria en guerre ${ }^{33}$ s'impose : comme le noyau dur de l'Etat libérien restait les blacks scorpios de Taylor, on assiste ici à un embryon d'Etat fonctionnant « à la violence », autorégulé par des phénomènes segmentaires de massacres factionnels et un pillage systématique qui n'hésite pas à recourir au travail forcé dans le secteur minier. Comme au Libéria, comme en Sierra Léone, le refus de l'Etat amène paradoxalement au revival de formes pré-étatiques, à une gouvernance ethniciste ou fondée sur la violence non ritualisée, mais en quelque sorte « expérimentale », dans le sens extrême du terme.

Dictature politico-militaire fortement appuyée de l'étranger, le « régime » de Bouaké se trouve très vulnérable devant des enquêtes à venir des ONG des droits de l'Homme et des tribunaux internationaux, qui, pour le moment, ont plus accès à la zone gouvernementale, si l'on admet qu'elles ne sont pas instrumentalisées. L'impunité sera, comme dans des post-conflits à la congolaise, un des enjeux de la fin de la rébellion.

Cependant, des éléments permettent à l'inverse de comprendre pourquoi la force des armes, l'exercice discontinu de la violence et la prédation érigée en système permettent une certaine gestion de la zone nord : l'appareil militaire se superpose en fait à un réseau puissant de communautés villageoises sénoufo et malinké, qui retrouvent de nouvelles fonctions d'autogestion, ou d'anciennes... car la situation rappelle l'éphémère «Empire nomade de l'Almamy Samory » dont se réclame implicitement le MPCI, tandis que les traditions locales se souviennent surtout de ses exactions.

33. Voir notre analyse générale: Galy M., « De la guerre nomade : sept approches du conflit autour de la Côte d'Ivoire », Cultures E Conflits, n55, pp. 163-196, Paris, L'Harmattan, 2004. 
Tout peut cependant arriver en Côte d'Ivoire, dont l'histoire récente montre bien les retournements d'alliances et de situations, en particulier une « internalisation » de la crise ${ }^{34}$ (par opposition à l'externationalisation actuelle et les manipulations élyséennes des institutions internationales contre le régime d'Abidjan), pourrait aboutir à une paradoxale " paix des braves». Des négociations ivoiro-ivoiriennes entre la rébellion et le pouvoir mettraient alors sur la touche les résolutions onusiennes, et la primature serait offerte à Guillaume Soro, renouvellant une voie très africaine des cadets revendiquant un pouvoir inaccessible par la force des armes.

A l'opposé, et comme depuis 2002, en cas d'échec du «dialogue direct » à condition de la neutralité des forces d'intervention au cas, peu probable, de leur aide à un "désarmement forcé », la reprise de la guerre paraitrait envisageable, une réunification du territoire permettant au régime actuel d'Abidjan d'aller en confiance aux élections. Pari qui semble toutefois bien risqué, et l'enlisement actuel paradoxalement plus sûr pour les deux parties.

Mais la fin du soutien matériel ou armé du Burkina, voire, en cas de changement de régime à Paris, la réduction par la force d'une nébuleuse chaotique de chefs de guerre divisés et de troupes démoralisées, est aussi envisageable. Alors, plutôt qu'une " anatomie » faudrait-il entrevoir les «métamorphoses » d'une rébellion ? Ou son autopsie ? Et si la réintégration des rebelles et de leur zone dans la République ivoirienne passait par une amnistie généralisée, cela rendrait-il nécessaire l'oubli de leurs crimes, à défaut de pardon ?

34. Selon la distinction que nous avons proposée : Galy M., «Qui gouverne la Côte-d'Ivoire ? Internalisation et internationalisation d'une crise politico-militaire », Politique étrangère, $\mathrm{n}^{\circ}$ 4/2005, pp. 795-808. 